\title{
PNEUMOPERITÔNEO PROGRESSIVO NO PRÉ-OPERATÓRIO DE HÉRNIAS INCISIONAIS VOLUMOSAS
}

\author{
PREOPERATIVE PNEUMOPERITONEUM IN THE MANAGEMENT OF GIANT \\ INCISIONAL HERNIAS
}

\author{
Diogo Franco Vieira de Oliveira, TCBC-RJ $^{1}$ \\ Luiz Fernando Fernandes Gonçalves, TCBC-RJ ${ }^{2}$ \\ César Silveira Cláudio-da-Silva, TCBC-RJ ${ }^{1}$ \\ Carlos Alberto Porchat, TCBC-RJ ${ }^{1}$ \\ Talita Franco, ECBC-RJ ${ }^{3}$
}

\begin{abstract}
RESUMO: Objetivo: Avaliar o pneumoperitôneo no preparo pré-operatório de pacientes portadores de hérnias incisionais volumosas. Método: Foram estudados cinco pacientes portadores de hérnias incisionais volumosas, encaminhados ao Serviço de Cirurgia Plástica do Hospital Universitário Clementino Fraga Filho da UFRJ, no período de agosto de 1994 a abril de 1997. Foi efetuado pneumoperitôneo progressivo, complementando a terapêutica pré-operatória instituída nestes pacientes. Resultados: Não houve intercorrências durante os procedimentos de instalação do pneumoperitôneo. Um paciente - o único do sexo masculino - não aceitou bem a expansão intra-abdominal e decidiu interromper o tratamento. As cirurgias se mostraram tecnicamente facilitadas: em três casos foi possível a sutura direta da aponeurose comprometida, sendo necessário o uso de tela de polipropileno em apenas um paciente. $O$ acompanhamento de 15 a 47 meses mostrou boa evolução e ausência de recidivas. Conclusão: O pneumoperitônio progressivo nos pacientes estudados, portadores de hérnias incisionais volumosas, se mostrou um método simples e eficaz do preparo pré-operatório.
\end{abstract}

Descritores: Pneumoperitônio; Cuidados pré-operatórios; Hérnia ventral.

\section{INTRODUÇÃO}

As grandes hérnias incisionais, além dos seus riscos inerentes de encarceramento e estrangulamento, causam deformidades importantes na superfície corporal, e alteram consideravelmente a relação conteúdo X continente da cavidade abdominal. Sua correção pode ser difícil, induzindo o cirurgião a utilizar métodos complementares para facilitar o procedimento cirúrgico, evitar complicações no período pósoperatório e diminuir o risco de recidivas.O pneumoperitôneo pré-operatório se mantém como uma boa opção. Em casos específicos, há uma melhora pronunciada das condições pré-operatórias dos pacientes e, conseqüentemente, de sua recuperação.

\section{MÉTODO}

No período de agosto de 1994 a abril de 1997, foram encaminhados ao Serviço de Cirurgia Plástica do Hospital Universitário da Universidade Federal do Rio de Janeiro cinco pacientes portadores de volumo-

1. Médico do Serviço de Cirurgia Plástica do Hospital Universitário Clementino Fraga Filho da Universidade Federal do Rio de Janeiro.

2. Doutorando do Departamento de Cirurgia da Faculdade de Medicina da Universidade Federal do Rio de Janeiro.

3. Chefe do Serviço de Cirurgia Plástica do Hospital Universitário Clementino Fraga Filho da Universidade Federal do Rio de Janeiro.

Recebido em: 16/01/2003

Aceito para publicação em: 03/06/2003

Trabalho realizado no Serviço de Cirurgia Plástica do Hospital Universitário Clementino Fraga Filho da Universidade F ederal do Rio de Janeiro. 

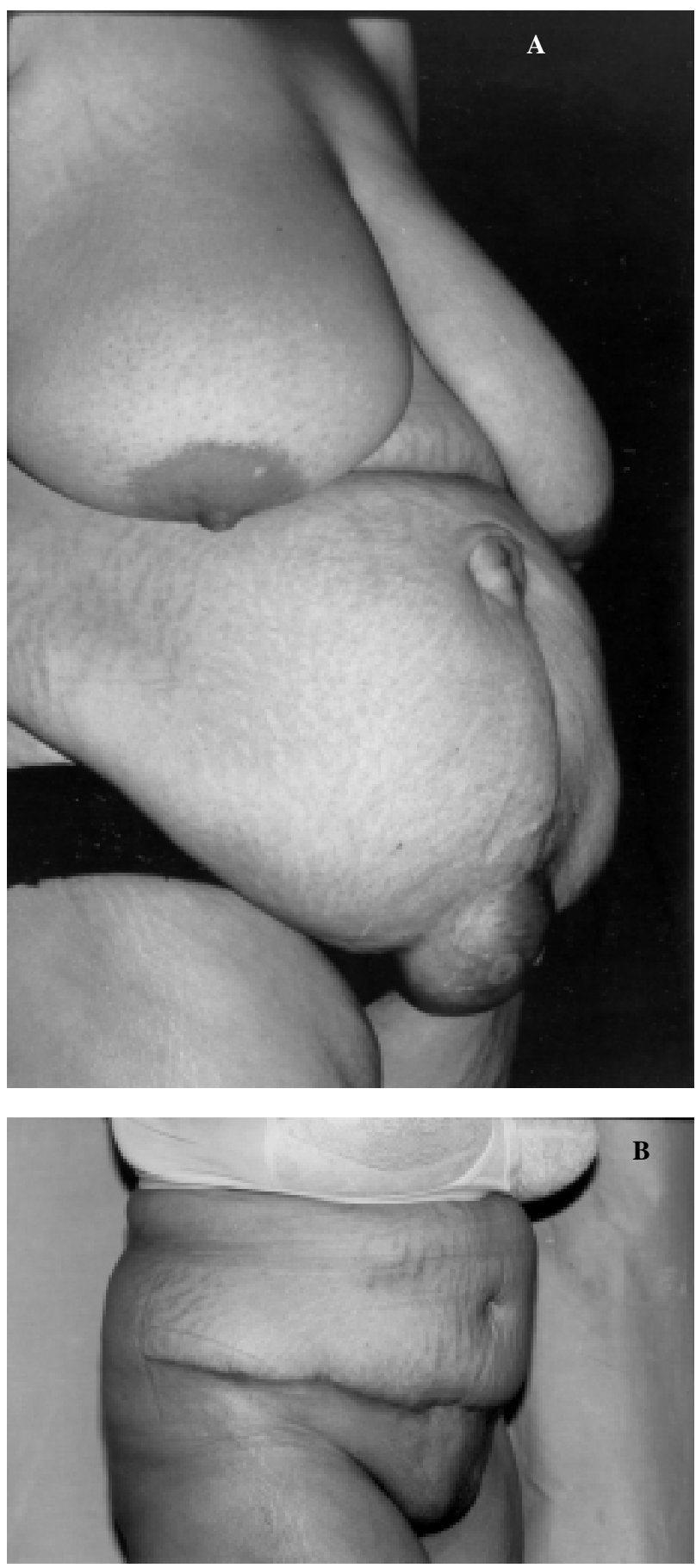

Figura 1 - A - Paciente apresentando extensa hérnia incisional, com partes encarceradas nos limites superior e inferior da cicatriz e extenso avental dermo-adiposo. B - Pós-operatório de 1 ano. Foifeita neo-onfaloplastia.

sas hérnias incisionais. Apresentavam área de comprometimento da parede abdominal de, pelo menos, 20 centímetros de diâmetro e a faixa etária situava-se entre 43 e 64 anos.
O único paciente do sexo masculino apresentava incisão mediana supra-umbilical devido à laparotomia exploradora por lesão por arma de fogo. Uma paciente tinha incisão semelhante (para correção de hérnia epigástrica) e, nas restantes, observava-se incisão mediana infra-umbilical para cesariana.

O pneumoperitôneo progressivo pré-operatório foi realizado por um período que variou de 13 a 20 dias, sendo iniciado logo após a internação do paciente. Através de punção percutânea, em condições assépticas e sob anestesia local, era introduzido na cavidade peritoneal "intracath" número 14, tendo sua extremidade fixada à pele através de sutura e curativo. O local de punção era determinado levando-se em consideração a apresentação da hérnia, com o intuito de se evitar acidentes de punção. Foi feita através do flanco esquerdo em três casos, do flanco direito em um caso e da cicatriz umbilical em um caso.

Utilizando seringa de $60 \mathrm{ml}$, era insuflado, diariamente, na cavidade peritoneal, um volume de ar atmosférico que, de acordo com a aceitação do paciente, oscilou entre 500 e $2500 \mathrm{ml}$. Sete dias após o inicio do procedimento, eram feitas radiografias de tórax e abdome. O volume total de ar insuflado variou entre 8700 a $14500 \mathrm{ml}$.

Para o ato cirúrgico utilizou-se via de acesso semelhante à de uma abdominoplastia convencional, complementada pela ressecção da cicatriz mediana já existente. Dessa forma eram confeccionados dois retalhos dermo-adiposos com pedículos superior e lateral, que conferiam bom acesso para a correção da hérnia. Em três pacientes foi possível a aproximação direta da aponeurose íntegra (Figura 1). Em apenas um caso foi necessária a utilização de tela de polipropileno para reconstrução da parede abdominal (Figura 2). O restante do procedimento assemelhava-se a uma abdominoplastia convencional com cicatriz transversa baixa. Em um caso, devido à presença prévia de cicatriz vertical supra-umbilical, a sutura final assemelhou-se a um " $T$ " invertido.

\section{RESULTADOS}

Os procedimentos de punção e insuflação ocorreram sem acidentes e foram bem aceitos por todos os pacientes, com exceção do único do sexo masculino. Este apresentou sudorese intensa, palidez e síncope após $100 \mathrm{ml}$ de ar insuflado. Interrompido o procedimento, houve melhora geral e o paciente optou por permanecer sem tratamento cirúrgico. 

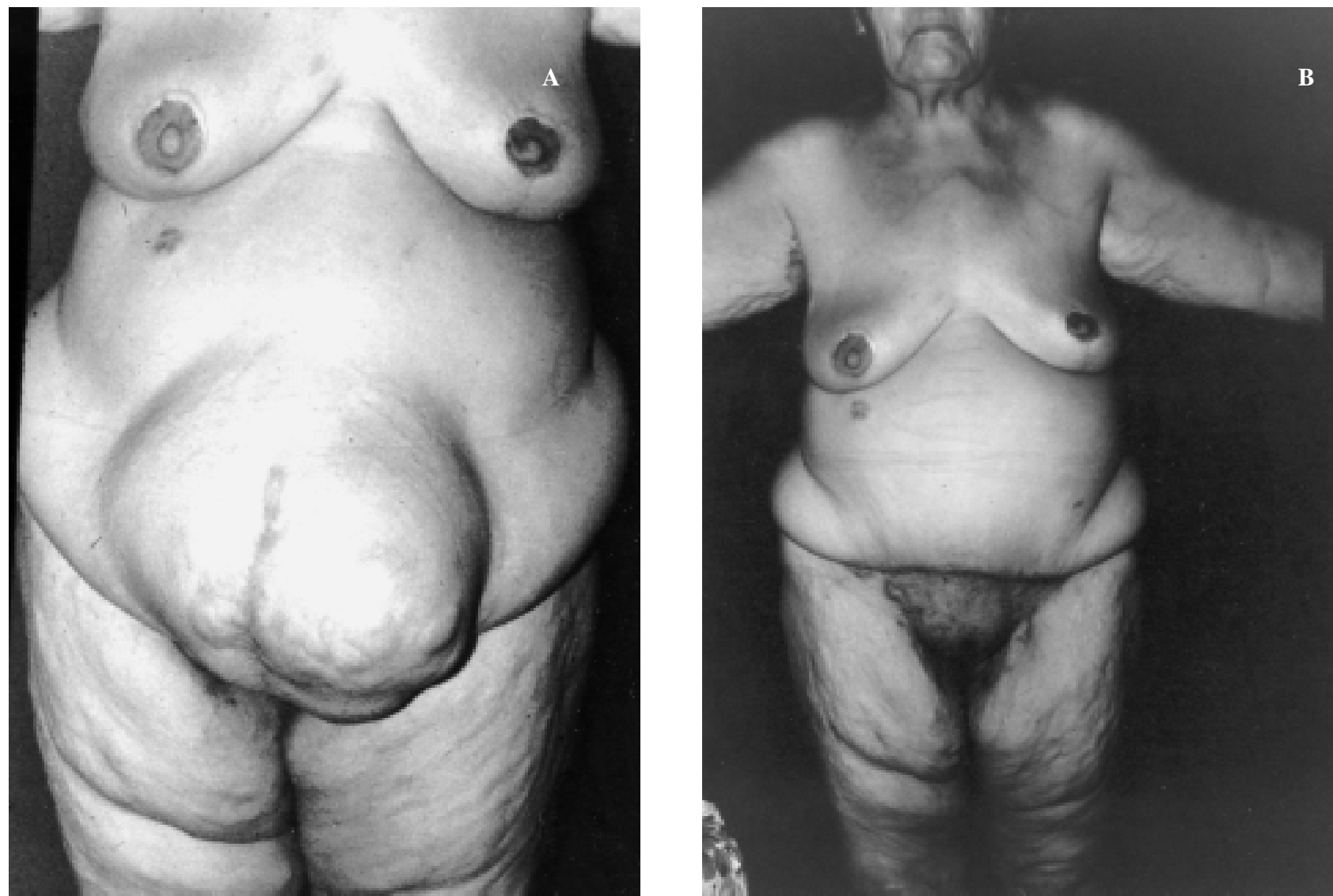

Figura 2 - A - Paciente apresentando volumosa hérnia através de cicatriz vertical mediana (cesarianas). B - Pós-operatório com 2 anos. A paciente se negou a ter o umbigo reconstruído.

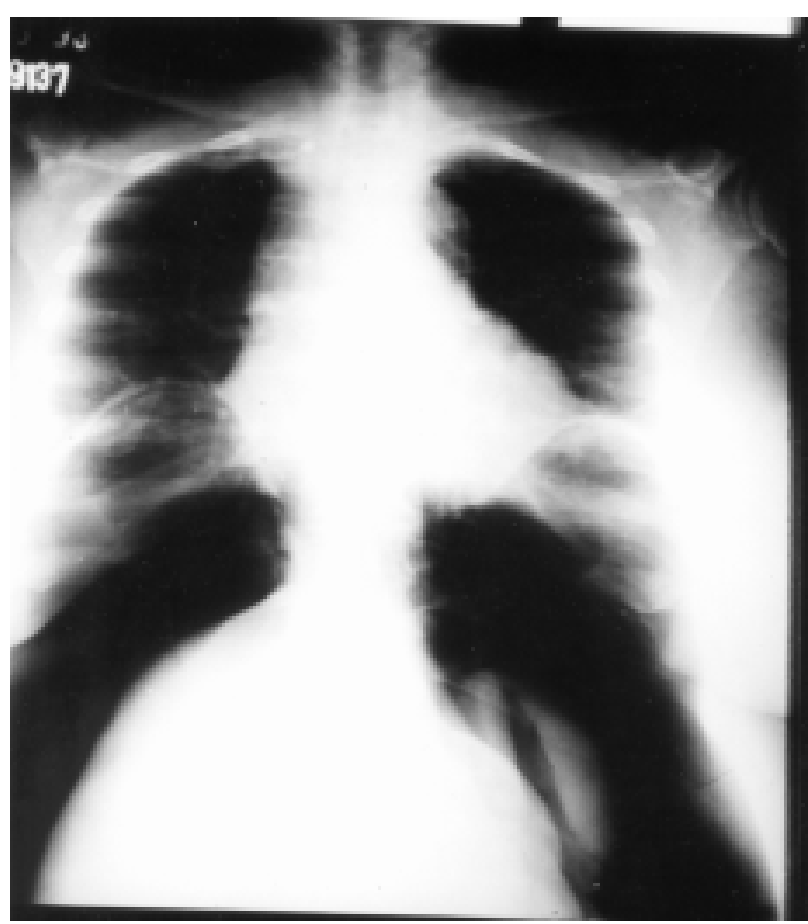

Figura 3 - Radiografia de controle pré-operatório auxiliando na quantificação do volume infiltrado.
As queixas mais comuns, durante a insuflação, foram de desconforto respiratório, pressão intra-abdominal e dor leve em região escapular, que cediam poucas horas após o procedimento.

As radiografias de controle pré-operatório permitiram avaliação da relação conteúdo $X$ continente da cavidade abdominal e do resultado do pneumoperitôneo realizado (Figura 3).

A paciente que apresentou cicatriz final em " $T$ " invertido evoluiu com sofrimento e necrose superficial de um segmento infra-umbilical, de aproximadamente $4 \mathrm{~cm}$ de largura. Este sofrimento se manifestou aos 10 dias de pós-operatório como epidermólise e secreção serosa. Após desbridamentos progressivos e curativos diários, houve completa epitelização.

Com um seguimento de 15 a 47 meses, não foram observadas recidivas das hérnias corrigidas.

\section{DISCUSSÃO}

As grandes hérnias incisionais causam retração das estruturas músculo-aponeuróticas ao seu 
redor, reduzindo a capacidade da cavidade abdominal e tornando impossível a redução definitiva do conteúdo herniário. Há, ainda, diminuição da atuação da musculatura abdominal acessória à respiração, além de comprometimento do retorno venoso. Este tipo de doença necessita preparo adequado a fim de se evitar complicações pós-operatórias e minimizar os riscos de recidiva.

Na década de 40, Goni Moreno relatou o uso e os benefícios do pneumoperitôneo progressivo préoperatório. Vários outros autores creditam a esse procedimento a importante redução das complicações pós-operatórias, do tempo cirúrgico e das recidivas. Isso, provavelmente, se deve à melhora das condições respiratórias e circulatórias, à expansão músculo-aponeurótica com aumento da capacidade volumétrica da cavidade abdominal, além da atividade adesiolítica, liberando aderências existentes ${ }^{1-7}$. Outra vantagem consiste no fato de que a tensão intraabdominal promove melhor delimitação do defeito facilitando a execução cirúrgica ${ }^{7}$. É difícil monitorizar, quantitativamente, a melhora observada, porém as evidências práticas são consistentes ${ }^{3}$.

A manutenção e a fixação do cateter possibilitam movimentação livre e evitam o desconforto da punção diária. Alguns autores utilizam insuflador para cirurgias vídeo-laparoscópicas, e gás carbônico ou óxido nitroso, para realização do pneumoperitôneo ${ }^{3,7}$, mas isso encarece o procedimento, além de ser necessário maior volume de insuflação, pois esses gases são mais rapidamente absorvidos $^{3}$.

As complicações são raras e geralmente sem gravidade (enfisema subcutâneo, pequeno hemoperitôneo etc.). A correção da doença e a aproximação da aponeurose sã se mostraram facilitadas e, em apenas um caso, foi necessária a utilização de tela de polipropileno para reconstrução da parede.

A maior desvantagem do pneumoperitôneo progressivo pré-operatório parece ser o tempo prolongado de internação e suas consequiências. A realização do procedimento a nível ambulatorial é possível $^{3}$, porém, nos últimos dias, a deambulação do paciente traz algum desconforto. Quanto à utilização de pneumoperitôneo progressivo peroperatório,seu efeito não é tão interessante ${ }^{7}$.

Sendo assim, acreditamos que a utilização do pneumoperitôneo progressivo pré-operatório é altamente recomendável para pacientes portadores de hérnias incisionais volumosas, tendo em vista a simplicidade e a inocuidade do procedimento, quando realizado com os devidos cuidados, e os benefícios advindos de sua utilização.

\begin{abstract}
Background:The authors study progressive pneumoperitoneum as preoperative management of patients with giant incisional hernias. Methods: Five patients suffering from giant incisional hernias treated at Plastic Surgery Service of the Hospital Universitário Clementino Fraga Filho of the Federal University of Rio de Janeiro, were studied. Before the surgical repair, progressive pneumoperitoneum was performed. Results: The procedure was uncomplicated, and improved surgical technique. In three cases repair was performed by simple aponeurotic plication. In one patient, the use of a prosthetic mesh was necessary to correct the abdominal defect. Another patient developed profuse sweating and faintness during the first session and refused further insufflation. The range of follow-up was 15-47 months and there was no recurrence. Conclusion: Progressive pneumoperitoneum was a simple and safe method to prepare patients with giant incisional hernias to surgery.
\end{abstract}

Key Words: Pneumoperitoneum; Preoperative care; Hernia, ventral.

\section{REFERÊNCIAS}

1. Micheau P, Grolleau JL - Eventration abdominale. Prise en charge et stratégie dans l'approche du futur opéré. Ann Chir Plast Esthet, 1999, 44(4): 325-338.
2. Micheau P, Grolleau JL, Rouge D - Grandes éventrations: clivage extensif profond de la paroi et prothèse. Presse Med,1995, 24(31): 1433-1437.

3. Caldironi MW, Romano M, Bozza F et al. - Progressive pneumoperitoneum in the managemament of giant 
incisional hernias: a study of 41 patients. Br J Surg, 1990,77(3): 306-307.

4. Coelho JC, Brenner AS, Freitas AT et al. - Progressive preoperative pneumoperitoneum in the repair of large abdominal hernias. Eur J Surg, 1993, 159(6-7): 339-341.

5. Ghiringhelli C, Ghiringhelli L, Redaelli G et al. - Derma versus rete nel trattamento dei laparoceli. Minerva Chir, 1992, 47(6): 419-422.

6. Masi C, Benvenuti P, Cardellicchio S et al. - Il pneumoperitoneo progressivo nella preparazione all'intervento chirurgico per voluminose ernie ventrali. Minerva Chir, 1992, 47(17): 1327-1335.
7. Winslet MC, Kumar V, Obeid ML - On-table pneumoperitoneum in the management of complicated incisional hernias. Ann R Coll Surg Engl, 1993, 75(3): $186-188$

Endereço para correspondência:

Praia de Botafogo 528 apto 1304-A

Rio de Janeiro. CEP 22250-040

Email:diogo@openlink.com.br 